Elsevier required licence: (c) 2017. This manuscript version is made available under the CC-BY-NC-ND 4.0 license http://creativecommons.org/licenses/by-nc-nd/4.0/ 
Baseline

\section{Newly discovered seagrass beds and their potential for blue carbon in the coastal seas of Hainan Island, South China Sea}

Zhijian Jiang $^{\text {a, b, c, } 1}$

Songlin Liüa, b, c, 1

Jingping Zhang ${ }^{\mathrm{a}, \mathrm{b}}$

Chunyu Zhao ${ }^{\mathrm{a}, \mathrm{b}, \mathrm{c}}$

Yunchao $\mathrm{Wu}^{\mathrm{a}, \mathrm{b}, \mathrm{c}}$

Shuo $\mathrm{Yu}^{\mathrm{a}, \mathrm{c}}$

Xia Zhang ${ }^{\mathrm{a}, \mathrm{c}}$

Chi Huang d, a

Xiaoping Huang ${ }^{\mathrm{a}, \mathrm{c}, *}$

xphuang@scsio.ac.cn

Manoj Kumar

${ }^{a}$ Key Laboratory of Tropical Marine Bio-resources and Ecology, South China Sea Institute of Oceanology, Chinese Academy of Sciences, Guangzhou 510301, PR China

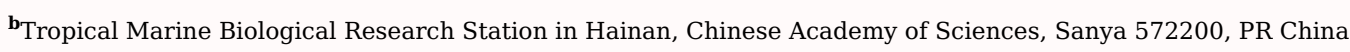

${ }^{\mathbf{c}}$ University of Chinese Academy of Sciences, Beijing 100049, PR China

${ }^{\mathbf{d}}$ College of Marine Life Science, Chinese Ocean University, Qingdao 266003, PR China

e Climate Change Cluster, Faculty of Science, University of Technology Sydney, Sydney, NSW 2007, Australia

*Corresponding author at: Key Laboratory of Tropical Marine Bio-resources and Ecology, South China Sea Institute of Oceanology, Chinese Academy of Sciences, Guangzhou 510301, PR China.

${ }^{\mathbf{1}}$ Author ZJ Jiang and SL Liu contribute equally to this article.

Abstract

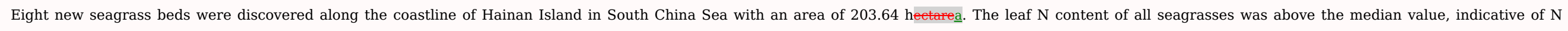

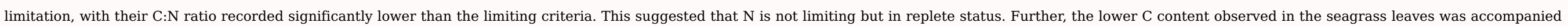

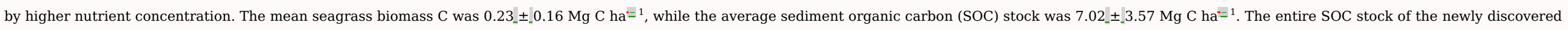

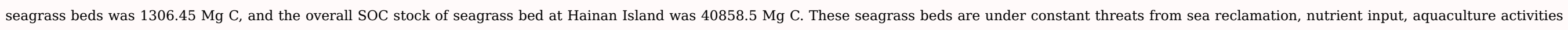
for oyster and snail farming, and fishing activities.

Keywords: Seagrass bed; Distribution; Nutrient dynamics; Habitat threats; Blue carbon; Management 


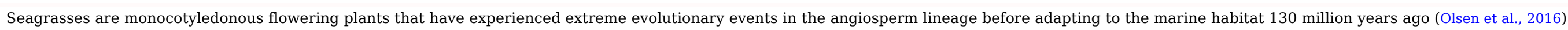

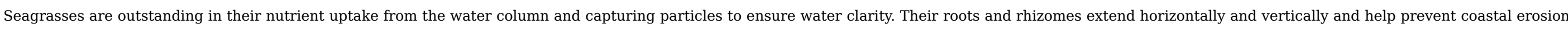

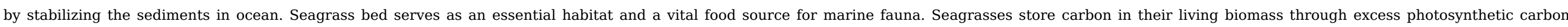

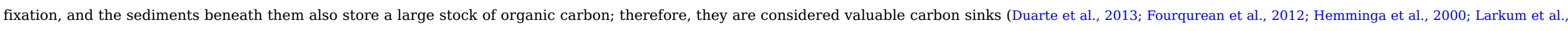
2006; Miyajima et al., 2015; Phang et al., 2015; Ricart et al., 2017; Waycott et al., 2009).

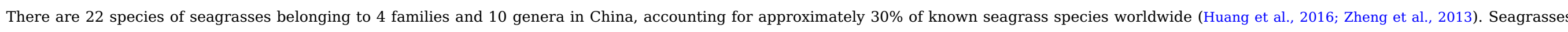

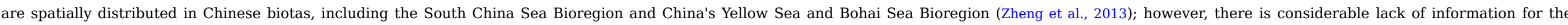

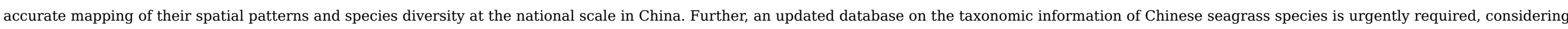

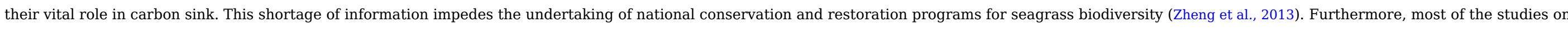

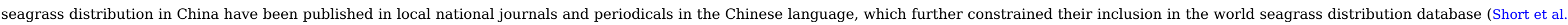
2007). Eventually, this has affected the contribution of Chinese seagrasses to the world seagrass carbon stock (Fourqurean et al., 2012).

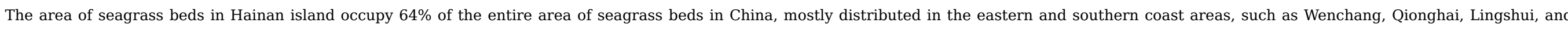

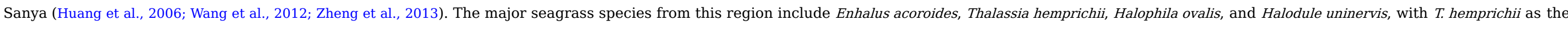

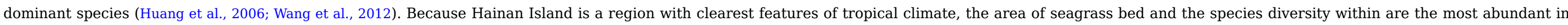

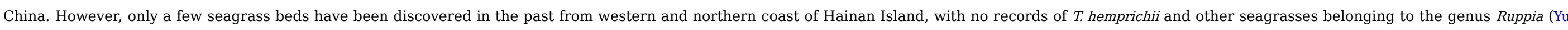

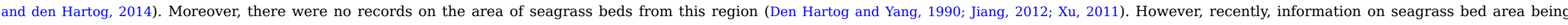

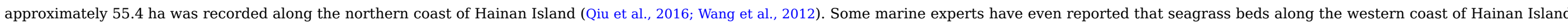

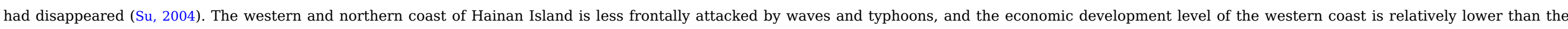

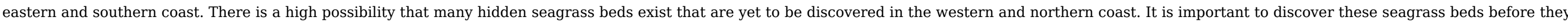

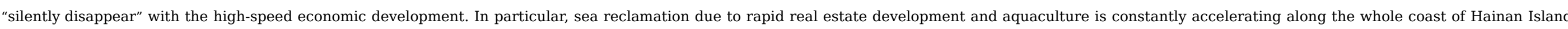

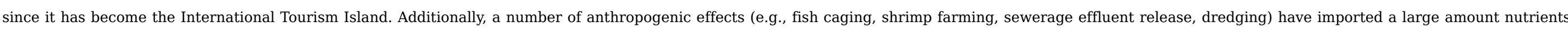

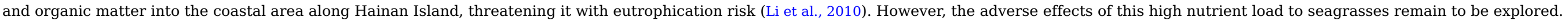

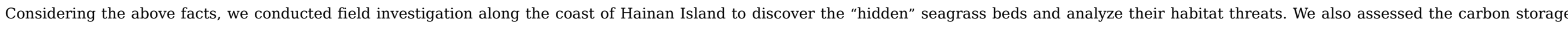

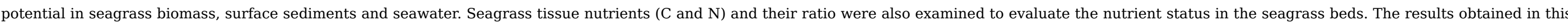

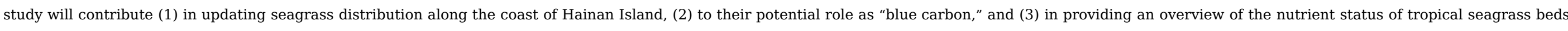
in the South China Sea region. It will strengthen the understanding that is needed to improve the management and protection of these ecologically and economically important marine floras.

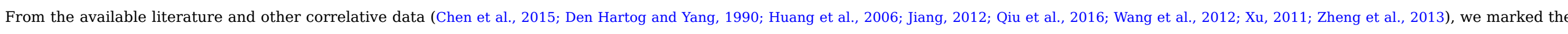

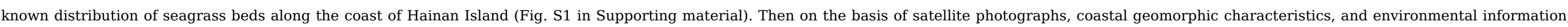

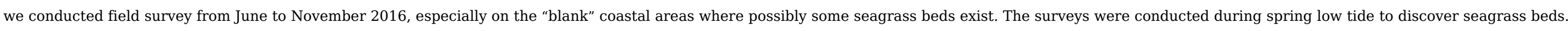

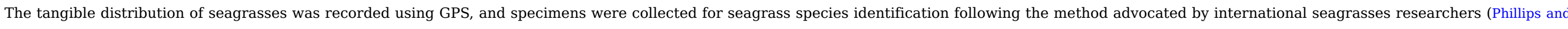

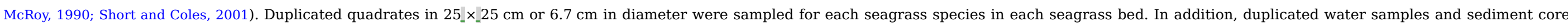

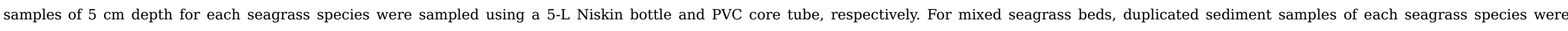
combined for analyzing the particle size.

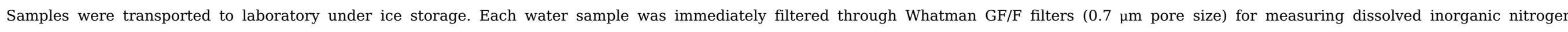

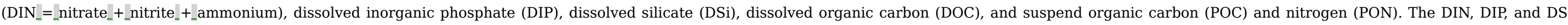

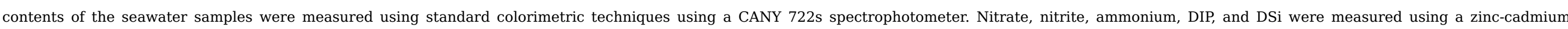




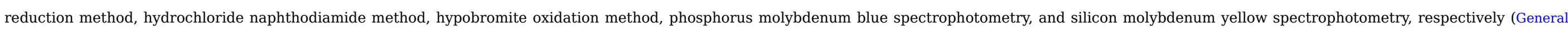

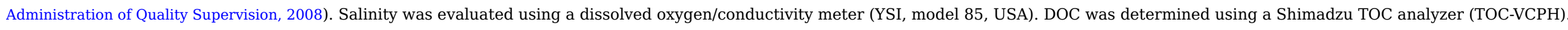

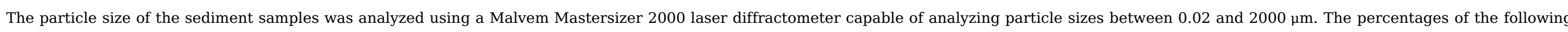

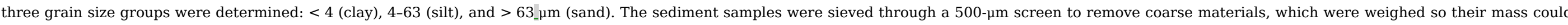

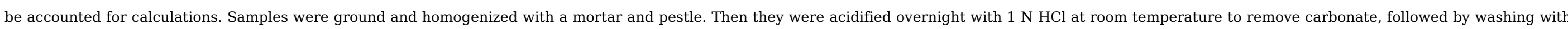

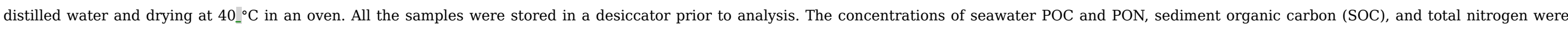
determined using a CHN Elemental Analyzer (Elementar, Vario EL-III, Germany)

We estimated the total vegetative carbon stock and total SOC stock $\left(\mathrm{C}_{\text {stock }}\right)$ of the top $5 \mathrm{~cm}$ of seagrass following the calculation (Howard et al., 2014):

Vegetative component carbon pool $(\mathrm{Mg} \mathrm{C} / \mathrm{ha})=$ Carbon content $\left(\mathrm{kg} \mathrm{C} / \mathrm{m}^{2}\right) \times(\mathrm{Mg} / 1000 \mathrm{~kg}) \times\left(10,000 \mathrm{~m}^{2} / \mathrm{ha}\right)$

Sediment carbon density $\left(\mathrm{g} / \mathrm{cm}^{3}\right)=$ dry bulk density $\left(\mathrm{g} / \mathrm{cm}^{3}\right) \times\left(\% \mathrm{C}_{\text {org }} / 100\right)$

Amount carbon in core section $\left(\mathrm{g} / \mathrm{cm}^{2}\right)=$ Sediment carbon density $\left(\mathrm{g} / \mathrm{cm}^{3}\right) \times$ thickness interval $(\mathrm{cm})$

Core carbon content $\left(\mathrm{g} / \mathrm{cm}^{2}\right)=$ Amount of carbon in core section A $\left(\mathrm{g} / \mathrm{cm}^{2}\right)+$ Amount of carbon in core section B $\left(\mathrm{g} / \mathrm{cm}^{2}\right)+$ Amount of carbon in core section C $\left(\mathrm{g} / \mathrm{cm}^{2}\right)+\ldots$ all the samples from a single core

Total core carbon $(\mathrm{Mg} \mathrm{C} / \mathrm{ha})=$ Summed core carbon $\left(\mathrm{g} / \mathrm{cm}^{2}\right) \times(1 \mathrm{Mg} / 1,000,000 \mathrm{~g}) \times\left(100,000,000 \mathrm{~cm}^{2} / 1 \mathrm{ha}\right)$

Average carbon in a core $=$ carbon content in core $1+$ carbon content in core $2+$ carbon content in core $3+\ldots . \mathrm{n} / \mathrm{n}$

Total organic carbon stock in a studied area $(\mathrm{Mg} \mathrm{C})=($ average core carbon from Statum A $(\mathrm{Mg} \mathrm{C} / \mathrm{ha}) \times$ area of Statum A (hectares) $)+($ average core carbon from Statum B $(\mathrm{Mg} \mathrm{C} / \mathrm{ha})) \times$ area of Statum B (hectares $)+\ldots$

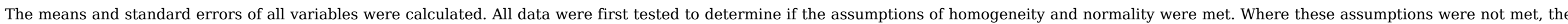

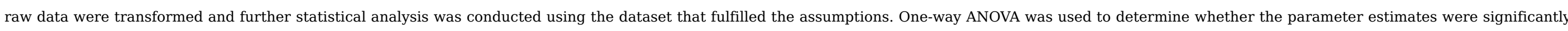

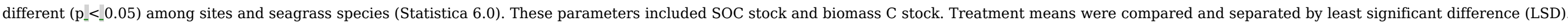

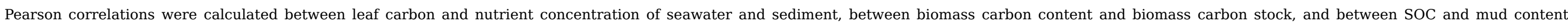
$\left(\% \_<0.63 \_\mu m\right)$.

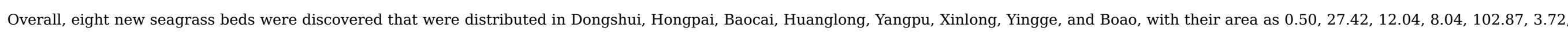

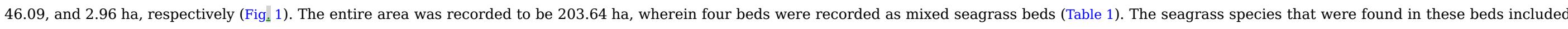

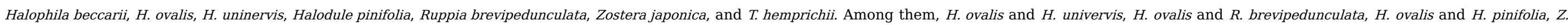
japonica and $H$. beccarii were mixed together in Hongpai, Xinlong, Yingge, Boao, respectively. H. ovalis and H. beccarii had a wider distribution along the coast of Hainan Island. 


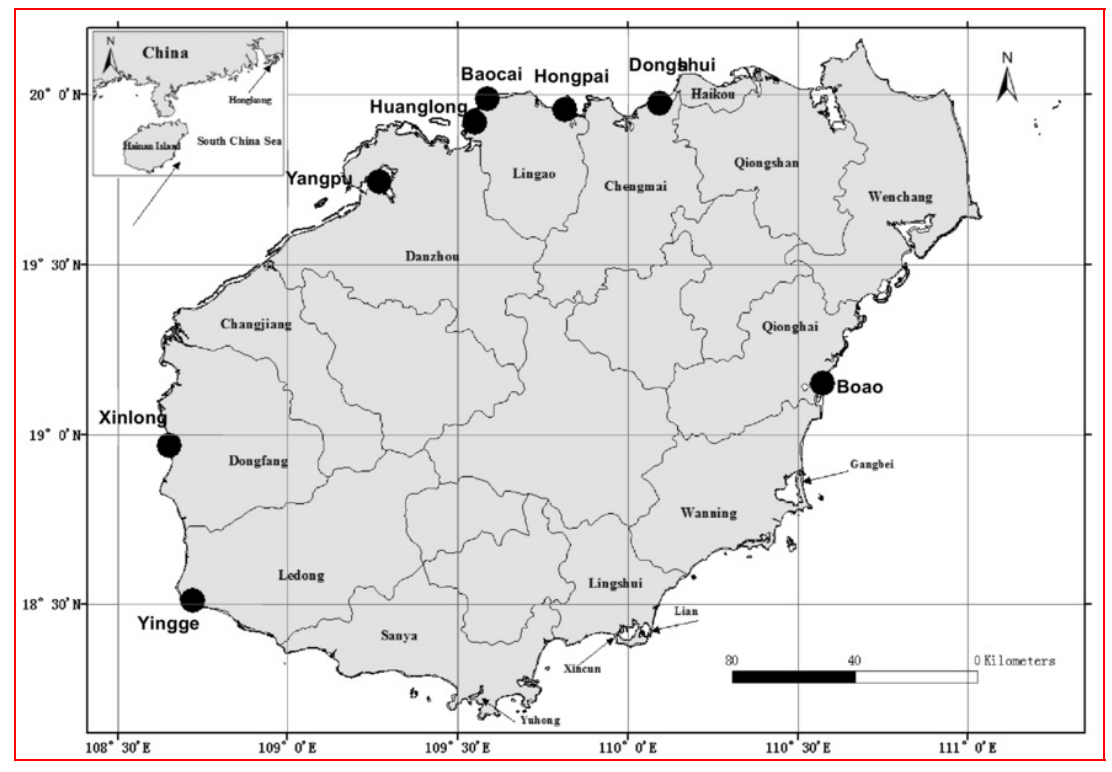

Fig. 1 Distribution of the newly discovered seagrass beds in the coastal seas of Hainan Island.

alt-text: Fig. 1

Table 1 Distribution, species, and area of newly discovered seagrass beds.

alt-text: Table 1

\begin{tabular}{|c|c|c|}
\hline Seagrass bed & Species & Area (ha) \\
\hline Dongshui & Halophila beccarii & 0.50 \\
\hline Hongpai & Halophila ovalis, Halodule uninervis & 27.42 \\
\hline Baocai & Thalassia hemprichii & 12.04 \\
\hline Huanglong & H. ovalis & 8.04 \\
\hline Yangpu & H. beccarii & 102.87 \\
\hline Xinlong & Ruppia brevipedunculata, H. ovalis & 3.72 \\
\hline Yingge & Halodule pinifolia, $H$. ovalis & 46.09 \\
\hline Boao & Zostera japonica, H. beccarii & 2.96 \\
\hline Total & & 203.64 \\
\hline
\end{tabular}

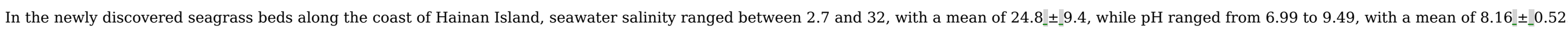

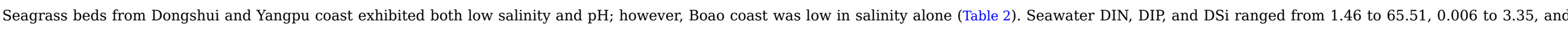

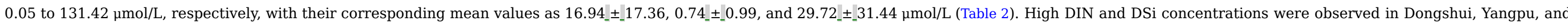

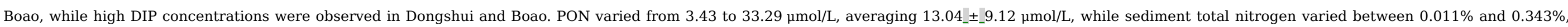
with a mean value at $0.082 \_ \pm 0.077 \%$ (Table 3 ). 
Table 2 Characteristics of seawater in newly discovered seagrass beds.

\begin{tabular}{|c|c|c|c|c|c|c|c|c|c|c|c|c|}
\hline Seagrass bed & Salinity & Temperature & $\mathrm{pH}$ & $\mathrm{NO}_{3}-\mathrm{N}(\mu \mathrm{mol} / \mathrm{L})$ & $\mathrm{NH}_{4}^{+}(\mu \mathrm{mol} / \mathrm{L})$ & $\mathrm{NO}_{2}-\mathrm{N}(\mu \mathrm{mol} / \mathrm{L})$ & DIN ( $\mu \mathrm{mol} / \mathrm{L})$ & DIP $(\mu \mathrm{mol} / \mathrm{L})$ & $\mathrm{DSi}(\mu \mathrm{mol} / \mathrm{L})$ & $\mathrm{DOC}(\mu \mathrm{mol} / \mathrm{L})$ & $\mathrm{POC}(\mu \mathrm{mol} / \mathrm{L})$ & PON $(\mu \mathrm{mol} / \mathrm{L})$ \\
\hline Dongshui & $15.75 \pm 0.35$ & $27.90 \pm 0.28$ & $7.75 \pm 0.01$ & $39.23 \pm 10.02$ & $16.87 \pm 3.54$ & $4.91 \pm 0.11$ & $61.01 \pm 6.37$ & $3.25 \pm 0.14$ & $46.54 \pm 1.91$ & 181 & $53.67 \pm 7.39$ & $9.83 \pm 2.54$ \\
\hline Hongpai & $29.50 \pm 0.71$ & $32.35 \pm 0.07$ & $8.50 \pm 0.15$ & $0.46 \pm 0.63$ & $2.26 \pm 1.14$ & - & $2.71 \pm 1.77$ & $0.83 \pm 0.09$ & $19.35 \pm 1.57$ & 212 & $94.26 \pm 41.99$ & $19.69 \pm 9.60$ \\
\hline Baocai & $31.90 \pm 0.14$ & $26.50 \pm 0.0$ & $8.01 \pm 0.01$ & $7.03 \pm 1.08$ & $7.13 \pm 2.46$ & - & $14.15 \pm 1.38$ & 0.260 .02 & $9.12 \pm 1.80$ & 254.7 & $22.67 \pm 1.11$ & $4.47 \pm 1.02$ \\
\hline Huanglong & $30.50 \pm 0.71$ & $26.00 \pm 0.71$ & $8.08 \pm 0.02$ & $2.90 \pm 0.81$ & $7.60 \pm 3.83$ & - & $10.50 \pm 4.64$ & $0.24 \pm 0.17$ & $16.16 \pm 0.32$ & 246.8 & $15.40 \pm 6.43$ & $18.36 \pm 21.12$ \\
\hline Yangpu & $20.50 \pm 0.71$ & $29.95 \pm 0.07$ & $7.72 \pm 0.04$ & $17.70 \pm 2.46$ & $7.62 \pm 0.08$ & $0.68 \pm 0.02$ & $25.99 \pm 1.25$ & $0.70 \pm 0.00$ & $34.58 \pm 7.51$ & $206.9 \pm 47.7$ & $73.53 \pm 5.11$ & $16.53 \pm 0.94$ \\
\hline Xinlong & $31.00 \pm 1.00$ & $32.97 \pm 0.81$ & $8.42 \pm 0.26$ & $2.46 \pm 2.08$ & $2.12 \pm 0.58$ & $0.03 \pm 0.02$ & $4.61 \pm 0.76$ & $0.05 \pm 0.07$ & $11.82 \pm 0.55$ & $258.3 \pm 75.6$ & $42.55 \pm 2.52$ & $9.41 \pm 2.37$ \\
\hline Yingge & $30.00 \pm 1.00$ & $30.63 \pm 0.57$ & $8.33 \pm 0.04$ & $3.68 \pm 2.32$ & $4.53 \pm 2.72$ & $0.09 \pm 0.04$ & $8.31 \pm 3.40$ & $0.11 \pm 0.03$ & $19.57 \pm 6.26$ & $562.1 \pm 169.3$ & $*$ & $*$ \\
\hline Boao & $3.80 \pm 1.56$ & $32.80 \pm 0.28$ & $8.24 \pm 1.77$ & $16.65 \pm 2.73$ & $3.76 \pm 3.49$ & $0.78 \pm 0.12$ & $21.20 \pm 6.35$ & $1.43 \pm 0.67$ & $107.59 \pm 33.70$ & $327.9 \pm 155.0$ & $*$ & $*$ \\
\hline
\end{tabular}

"-" indicates below detection limit, “*” indicates data missing.

Table 3 Characteristics of sediments in newly discovered seagrass beds.

alt-text: Table 3

\begin{tabular}{|c|c|c|c|c|c|c|c|}
\hline \multirow[t]{2}{*}{ Seagrass bed } & \multirow[t]{2}{*}{ Water content (\%) } & \multirow[t]{2}{*}{ Bulk density $\left(\mathrm{g} / \mathrm{cm}^{3}\right)$} & \multicolumn{3}{|c|}{ Sediment particle sizes } & \multirow[t]{2}{*}{ Carbon (\%) } & \multirow[t]{2}{*}{ Nitrogen (\%) } \\
\hline & & & Sand (\%) & Silt (\%) & Clay (\%) & & \\
\hline Dongshui & $21.76 \pm 0.46$ & $1.86 \pm 0.26$ & $79.64 \pm 3.24$ & $16.55 \pm 2.70$ & $3.81 \pm 0.55$ & $1.20 \pm 0.17$ & $0.244 \pm 0.141$ \\
\hline Hongpai & $27.50 \pm 2.86$ & $1.52 \pm 0.16$ & $98.16 \pm 2.60$ & $0.78 \pm 1.10$ & $1.06 \pm 1.50$ & $1.50 \pm 0.31$ & $0.012 \pm 0.001$ \\
\hline Baocai & $27.37 \pm 2.92$ & $1.40 \pm 0.05$ & $99.32 \pm 0.96$ & $0.68 \pm 0.96$ & $0.00 \pm 0.00$ & $0.33 \pm 0.08$ & $0.031 \pm 0.008$ \\
\hline Huanglong & $58.88 \pm 4.63$ & $1.15 \pm 0.09$ & $40.14 \pm 5.13$ & $41.94 \pm 4.46$ & $17.92 \pm 0.33$ & $1.15 \pm 0.08$ & $0.131 \pm 0.002$ \\
\hline Yangpu & $20.43 \pm 3.85$ & $1.69 \pm 0.04$ & $81.52 \pm 8.50$ & $13.25 \pm 4.06$ & $5.24 \pm 4.44$ & $0.51 \pm 0.23$ & $0.051 \pm 0.019$ \\
\hline Xinlong & $43.89 \pm 7.07$ & $1.29 \pm 0.11$ & $34.23 \pm 30.66$ & $50.17 \pm 21.53$ & $15.60 \pm 9.13$ & $0.87 \pm 0.11$ & $0.098 \pm 0.017$ \\
\hline Yingge & $38.27 \pm 1.77$ & $1.44 \pm 0.05$ & $58.65 \pm 8.42$ & $27.36 \pm 5.39$ & $13.99 \pm 3.03$ & $1.30 \pm 0.37$ & $0.102 \pm 0.058$ \\
\hline Boao & $26.30 \pm 4.23$ & $1.53 \pm 0.11$ & $80.77 \pm 11.15$ & $14.12 \pm 4.19$ & $5.11 \pm 3.12$ & $0.39 \pm 0.21$ & $0.046 \pm 0.035$ \\
\hline
\end{tabular}

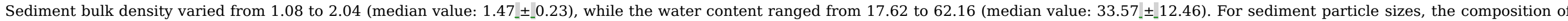

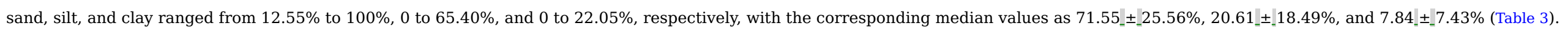

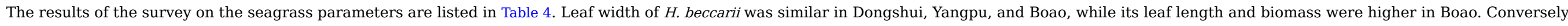

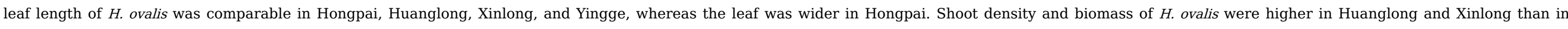
Hongpai and Yingge. Leaf length of $H$. pinifolia was longer than that of $H$. uninervis. Interestingly, seeds of $R$. brevipedunculata and $H$. ovalis were also observed on their plants.

Table 4 Characteristics of seagrass in newly discovered seagrass beds. 


\begin{tabular}{|c|c|c|c|c|c|c|c|c|c|c|c|c|c|c|}
\hline \multirow{2}{*}{$\begin{array}{c}\text { Seagrass } \\
\text { bed }\end{array}$} & \multirow[t]{2}{*}{ Species } & \multirow{2}{*}{$\begin{array}{l}\text { Leaf length } \\
\quad(\mathrm{cm})\end{array}$} & \multirow{2}{*}{$\begin{array}{l}\text { Leaf width } \\
\quad(\mathrm{cm})\end{array}$} & \multirow{2}{*}{$\begin{array}{l}\text { Shoot density } \\
\left(\text { shoots } / \mathrm{m}^{2}\right)\end{array}$} & \multirow{2}{*}{$\begin{array}{c}\text { Aboveground } \\
\text { biomass (DW } \\
\left.\mathrm{g} / \mathrm{m}^{2}\right)\end{array}$} & \multirow{2}{*}{$\begin{array}{l}\text { Belowground } \\
\text { biomass (DW } \\
\left.\mathrm{g} / \mathrm{m}^{2}\right)\end{array}$} & \multicolumn{4}{|c|}{ Aboveground tissue } & \multicolumn{4}{|c|}{ Belowground tissue } \\
\hline & & & & & & & $\mathrm{C} \%$ & N\% & $\mathrm{C} / \mathrm{N}$ ratio & $\begin{array}{c}\text { Biomass C } \\
\left(\mathrm{Mg} \mathrm{C} \mathrm{ha-}^{-1}\right)\end{array}$ & $\mathrm{C} \%$ & N\% & $\mathrm{C} / \mathrm{N}$ ratio & $\begin{array}{l}\text { Bioma } \\
(\mathrm{Mg} \mathrm{C}\end{array}$ \\
\hline Dongshui & $\begin{array}{l}\text { Halophila } \\
\text { beccarii }\end{array}$ & $0.79 \pm 0.11$ & $0.16 \pm 0.03$ & $7833 \pm 1650$ & $22.14 \pm 6.92$ & $19.79 \pm 6.63$ & $30.6 \pm 0.8$ & $3.0 \pm 0.2$ & $10.2 \pm 0.3$ & $0.068 \pm 0.023$ & $35.1 \pm 7.2$ & $2.3 \pm 0.2$ & $15.5 \pm 1.7$ & $0.067 \pm$ \\
\hline \multirow[t]{2}{*}{ Hongpai } & Halophila ovalis & $2.00 \pm 0.54$ & $1.20 \pm 0.38$ & $5850 \pm 1344$ & $35.75 \pm 28.14$ & $22.38 \pm 17.47$ & $39.3 \pm 12.9$ & $3.0 \pm 0.0$ & $13.3 \pm 4.5$ & $0.159 \pm 0.157$ & $25.1 \pm 2.9$ & $1.0 \pm 0.1$ & $26.9 \pm 7.1$ & $0.059 \pm$ \\
\hline & $\begin{array}{l}\text { Halodule } \\
\text { uninervis }\end{array}$ & $3.17 \pm 1.70$ & $0.09 \pm 0.01$ & $4233 \pm 2216$ & $13.59 \pm 3.01$ & $29.03 \pm 14.75$ & $38.3 \pm 11.4$ & $2.7 \pm 0.7$ & $13.9 \pm 0.4$ & $0.050 \pm 0.004$ & $35.0 \pm 1.0$ & $1.8 \pm 1.1$ & $24.3 \pm 15.3$ & $0.101 \pm$ \\
\hline Baocai & $\begin{array}{l}\text { Thalassia } \\
\text { hemprichii }\end{array}$ & $8.75 \pm 2.59$ & $0.82 \pm 0.13$ & $448 \pm 136$ & $26.02 \pm 8.14$ & $161.01 \pm 46.12$ & $34.8 \pm 0.7$ & $2.6 \pm 0.1$ & $13.2 \pm 0.5$ & $0.090 \pm 0.027$ & $31.5 \pm 1.2$ & $1.6 \pm 0.2$ & $19.5 \pm 1.4$ & $0.510 \pm$ \\
\hline Huanglong & Halophila ovalis & $1.76 \pm 0.13$ & $0.71 \pm 0.08$ & $12924 \pm 1081$ & $51.92 \pm 4.52$ & $45.18 \pm 9.79$ & $33.1 \pm 2.5$ & $3.4 \pm 0.3$ & $9.8 \pm 0.1$ & $0.172 \pm 0.028$ & $24.2 \pm 0.8$ & $1.6 \pm 0.4$ & $15.7 \pm 3.6$ & $0.110 \pm$ \\
\hline Yangpu & $\begin{array}{l}\text { Halophila } \\
\text { beccarii }\end{array}$ & $0.52 \pm 0.14$ & $0.13 \pm 0.03$ & $12500 \pm 3535$ & $15.11 \pm 11.50$ & $16.30 \pm 11.70$ & $32.2 \pm 2.7$ & $3.0 \pm 0.2$ & $10.7 \pm 0.4$ & $0.050 \pm 0.041$ & $36.5 \pm 1.7$ & $1.6 \pm 0.0$ & $22.4 \pm 1.3$ & $0.060 \pm$ \\
\hline \multirow[t]{2}{*}{ Xinlong } & $\begin{array}{l}\text { Ruppia } \\
\text { brevipedunculata }\end{array}$ & $5.63 \pm 1.53$ & $0.04 \pm 0.01$ & $11900 \pm 2970$ & $41.56 \pm 1.85$ & $19.04 \pm 1.59$ & $36.9 \pm 1.8$ & $2.6 \pm 0.6$ & $14.7 \pm 2.6$ & $0.154 \pm 0.001$ & $35.6 \pm 4.0$ & $1.5 \pm 0.1$ & $23.6 \pm 5.0$ & $0.068 \pm$ \\
\hline & Halophila ovalis & $1.89 \pm 0.27$ & $0.75 \pm 0.13$ & $11200 \pm 4808$ & $45.21 \pm 36.98$ & $54.93 \pm 23.33$ & $32.4 \pm 1.1$ & $2.7 \pm 0.2$ & $12.2 \pm 0.6$ & $0.149 \pm 0.125$ & $30.3 \pm 1.1$ & $1.6 \pm 0.0$ & $18.8 \pm 0.6$ & $0.168 \pm$ \\
\hline \multirow[t]{2}{*}{ Yingge } & $\begin{array}{l}\text { Halodule } \\
\text { pinifolia }\end{array}$ & $6.94 \pm 1.88$ & $0.10 \pm 0.01$ & $4675 \pm 601$ & $29.44 \pm 0.19$ & $28.07 \pm 0.99$ & $34.5 \pm 2.8$ & $2.8 \pm 0.2$ & $12.2 \pm 0.0$ & $0.101 \pm 0.008$ & $31.7 \pm 1.0$ & $2.3 \pm 0.1$ & $14.0 \pm 0.0$ & $0.089 \pm$ \\
\hline & Halophila ovalis & $1.66 \pm 0.28$ & $0.65 \pm 0.10$ & $6850 \pm 919$ & $11.65 \pm 1.02$ & $10.13 \pm 1.90$ & $29.6 \pm 0.4$ & $2.8 \pm 0.0$ & $10.4 \pm 0.1$ & $0.035 \pm 0.003$ & $23.9 \pm 2.7$ & $1.3 \pm 0.2$ & $18.0 \pm 0.5$ & $0.024 \pm$ \\
\hline \multirow[t]{2}{*}{ Boao } & Zostera japonica & $21.73 \pm 3.15$ & $0.17 \pm 0.04$ & $2733 \pm 450$ & $65.52 \pm 7.48$ & $14.68 \pm 0.62$ & $32.1 \pm 0.7$ & $2.2 \pm 0.1$ & $10.2 \pm 0.6$ & $0.202 \pm 0.016$ & $34.9 \pm 0.1$ & $1.3 \pm 0.2$ & $19.2 \pm 0.5$ & $0.052 \pm$ \\
\hline & $\begin{array}{l}\text { Halophila } \\
\text { beccarii }\end{array}$ & $1.26 \pm 0.34$ & $0.17 \pm 0.04$ & $10200 \pm 3111$ & $30.90 \pm 12.08$ & $42.54 \pm 35.33$ & $34.5 \pm 1.7$ & $3.4 \pm 0.4$ & $14.5 \pm 0.4$ & $0.109 \pm 0.049$ & $33.2 \pm 1.2$ & $1.7 \pm 0.0$ & $27.8 \pm 3.8$ & $0.143 \pm$ \\
\hline
\end{tabular}

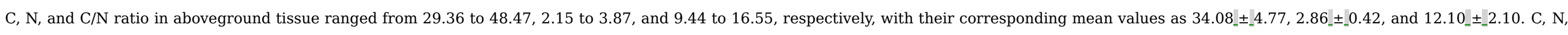

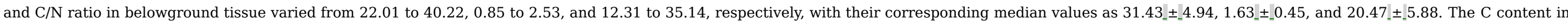

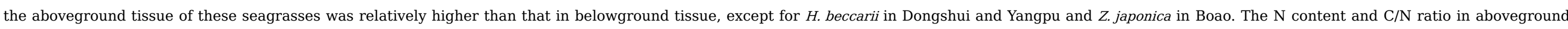

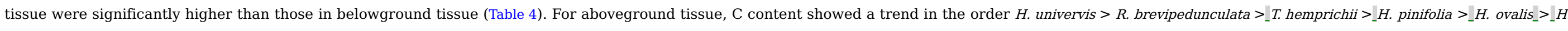

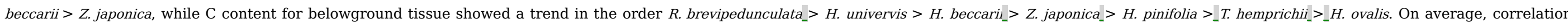

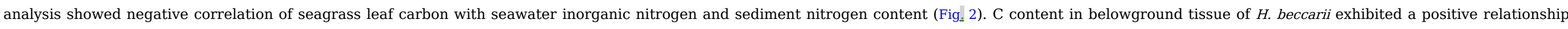

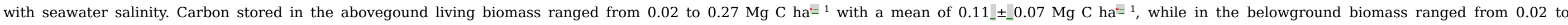

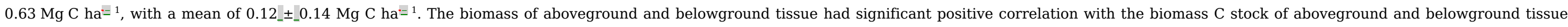

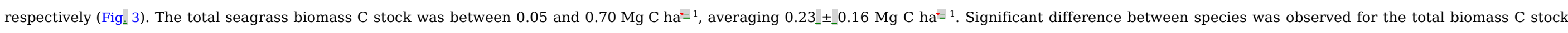
$\left(\mathrm{F}_{-}={ }_{-} 3.615, \mathrm{p}_{-}<0.05\right)$, with a trend in the order T. hemprichil $>$ Z. japonica_ $>$ R. brevipedunculata $>$ H. ovalis_> H. pinifolia_> H. beccarii_> H. uninervis $($ Fig. 4$)$. 

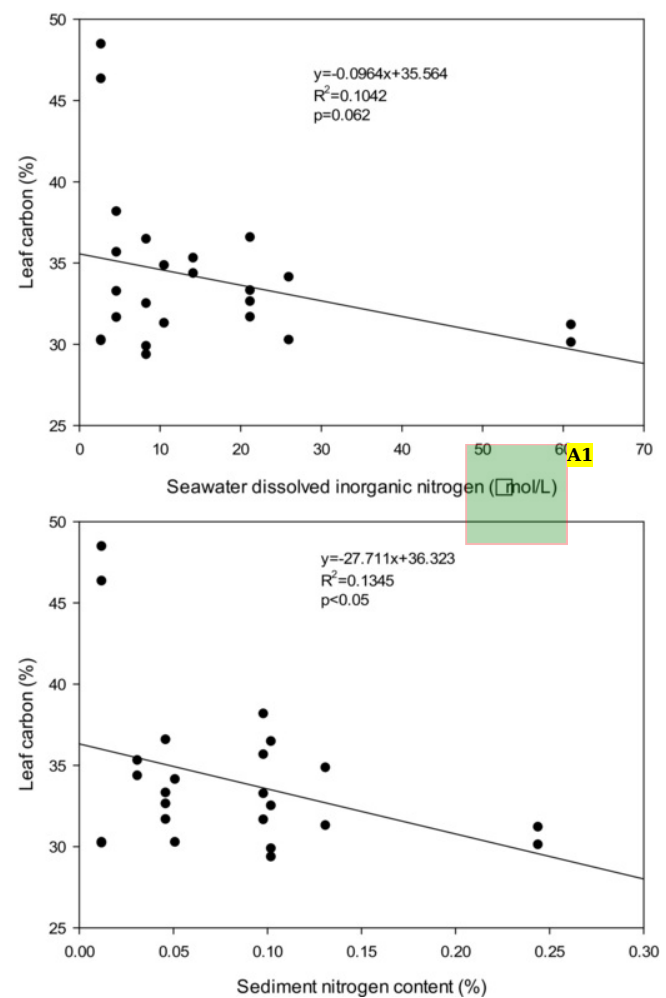

Fig. 2 Relationship between seagrass leaf carbon and seawater dissolved inorganic and sediment nitrogen in newly discovered seagrass beds in the coastal seas of Hainan Island alt-text: Fig. 2 Annotations:

A1. $(\mu \mathrm{mol} / \mathrm{L})$ 

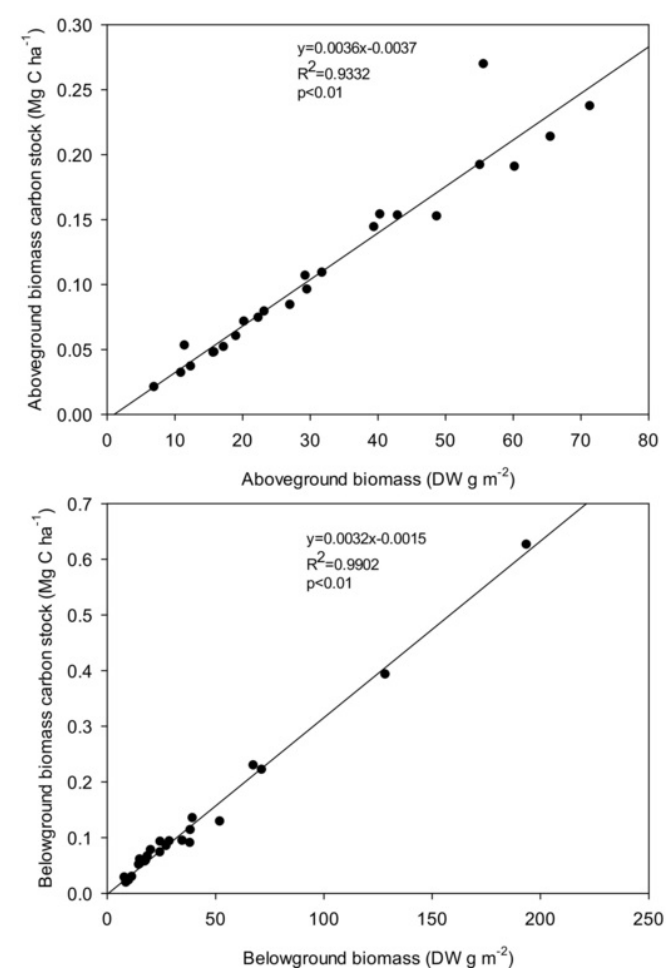

Fig. 3 Relationship between the biomass of aboveground and belowground tissue and biomass carbon stock

alt-text: Fig. 3

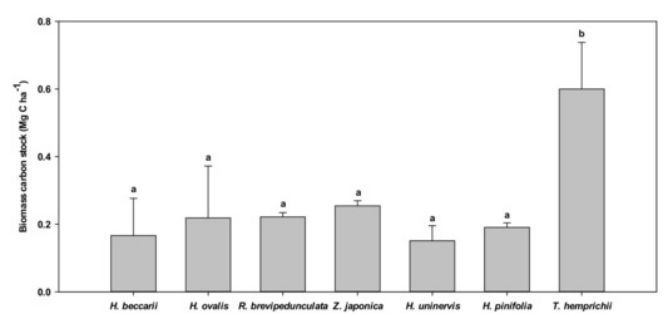

Fig. 4 Biomass carbon stock of different seagrass species in the coastal seas of Hainan Island.

alt-text: Fig. 4

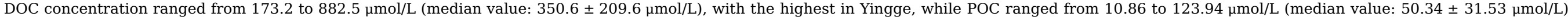

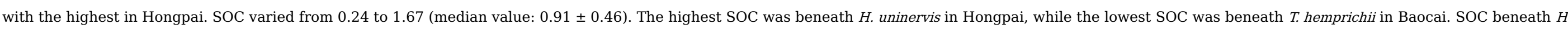

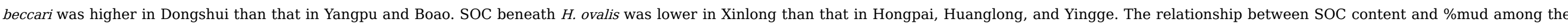
newly discovered seagrass beds is shown in Fig. 5. SOC and mud contents were only slightly positively correlated, albeit insignificantly. 


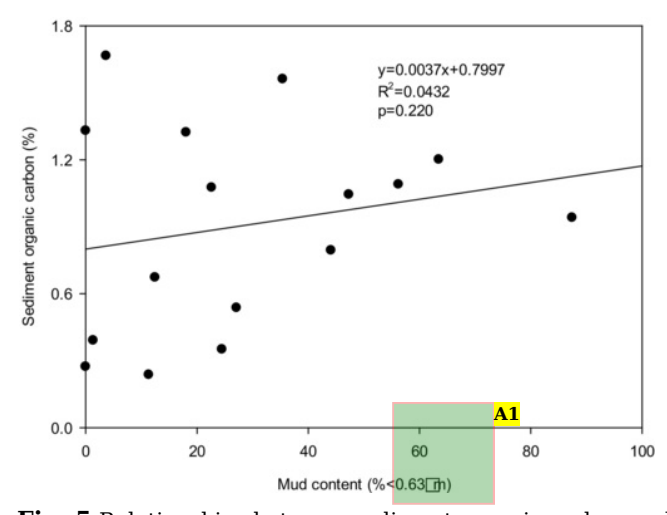

Fig. 5 Relationships between sediment organic carbon and mud content $(\%<0.63 \mu \mathrm{m})$ in the newly discovered seagrass beds in the coastal seas of Hainan Island.

alt-text: Fig. 5

Annotations:

A1. $(\%<0.63 \mu \mathrm{m})$

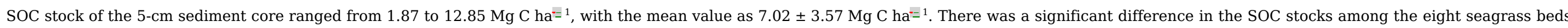

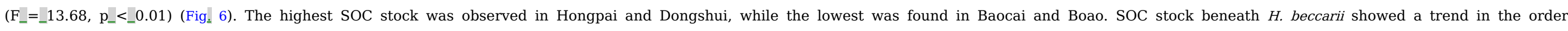
Hongpail> Yangpu_> Boao.

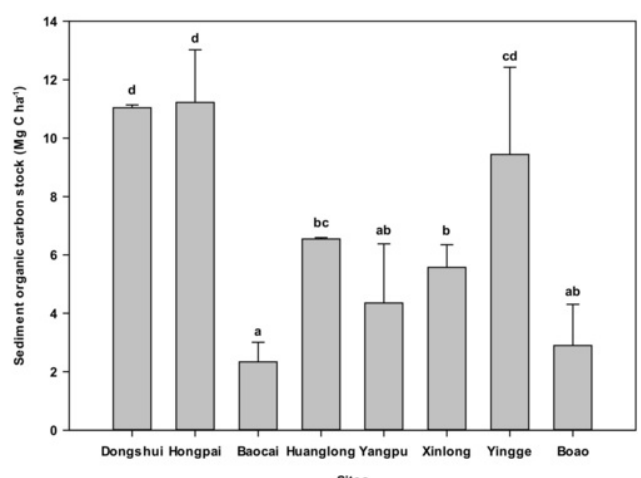

Fig. 6.Fig. 6 Sediment organic carbon stock in newly discovered seagrass beds in the coastal seas of Hainan Island

\section{alt-text: Fig. 6}

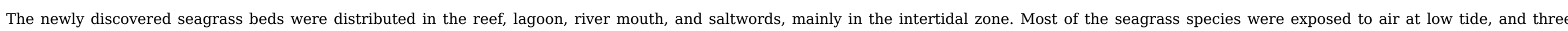

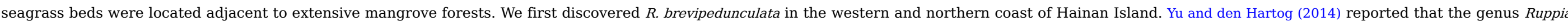

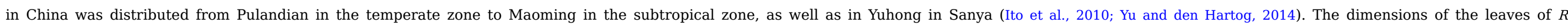

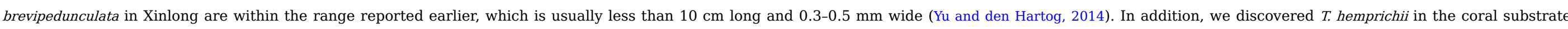

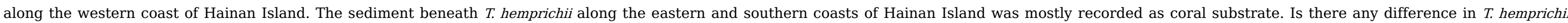

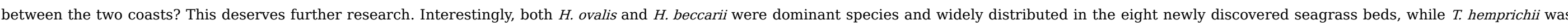

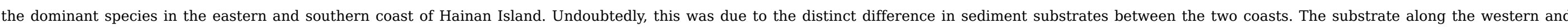




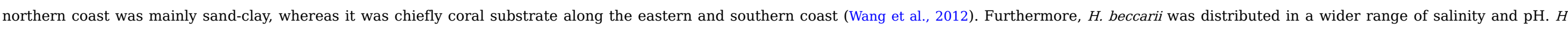

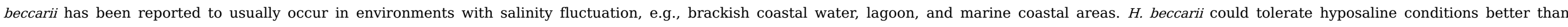

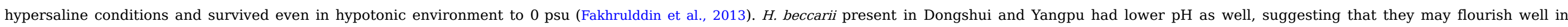
futuristic oceans conditions.

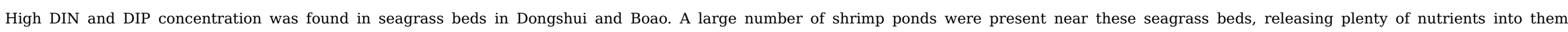

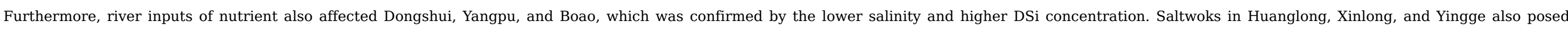

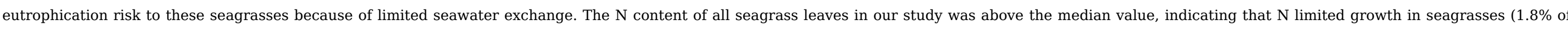

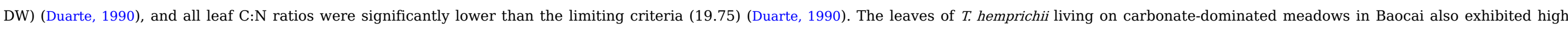

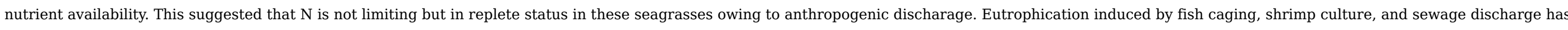

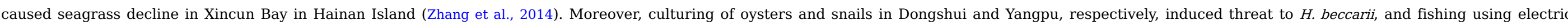

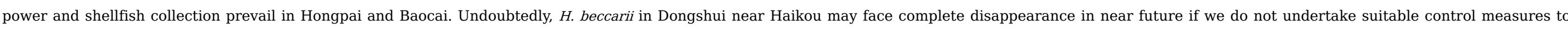

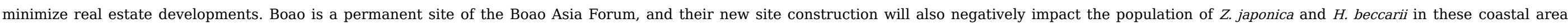

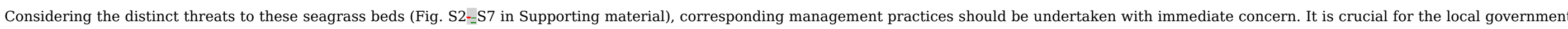

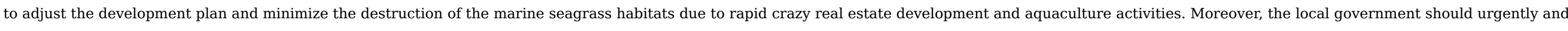
comprehensively control the nutrient loading to the nearshore environment along the coast of Hainan Island to keep the important seagrass resources healthy.

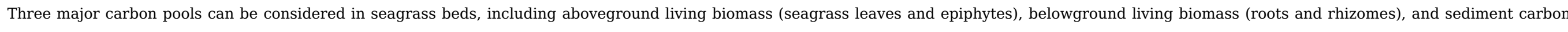

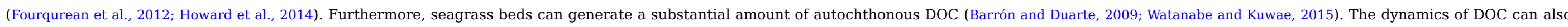

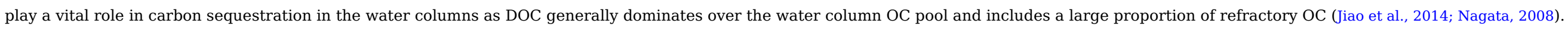

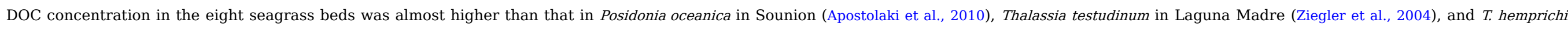

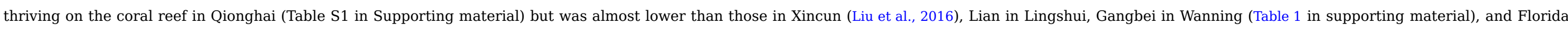

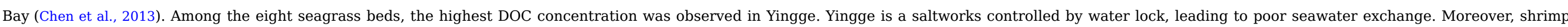

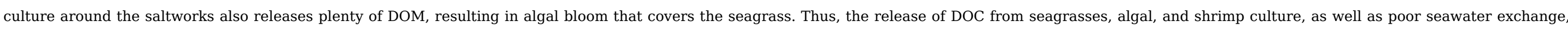

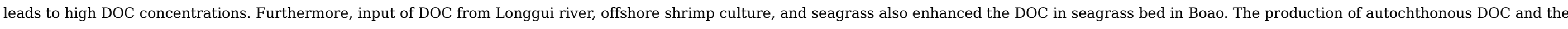

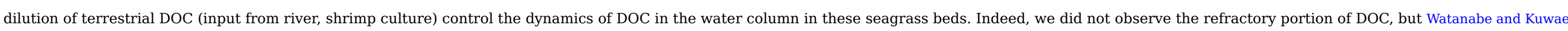
(2015) found that seagrass directly produces refractory DOM preserved in the water column.

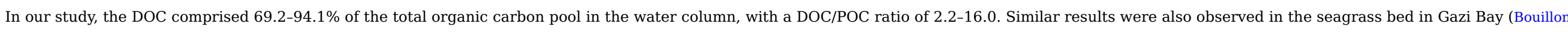

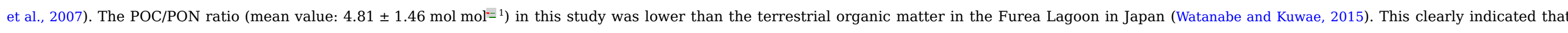

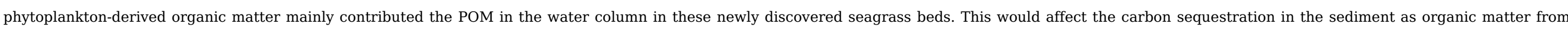
phytoplankton was less efficiently preserved than organic matter from terristrial input and seagrass (Watanabe and Kuwae, 2015).

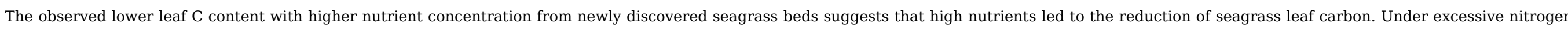

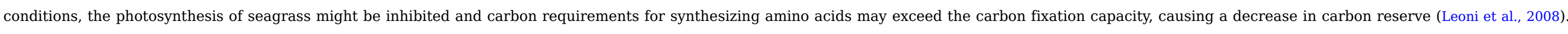

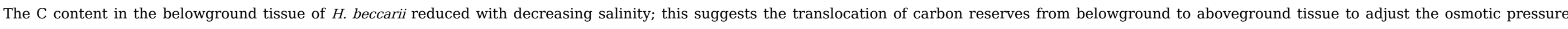

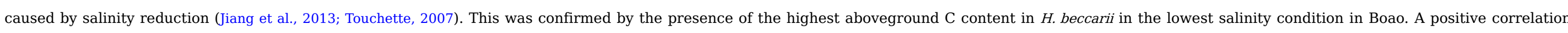

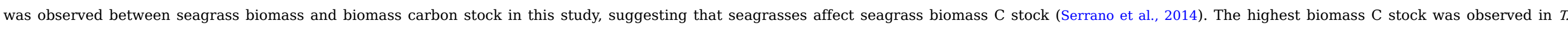

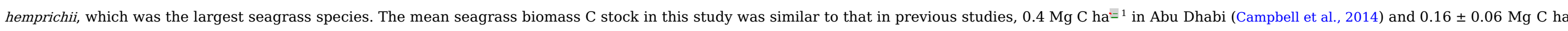

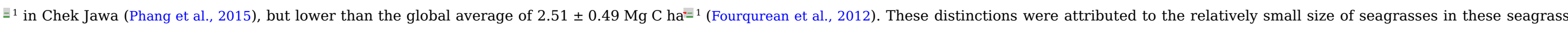




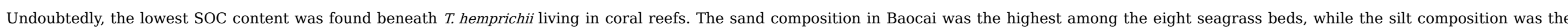

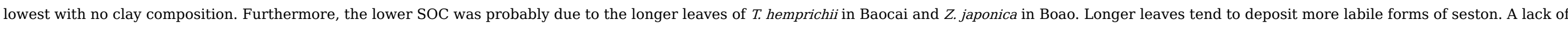

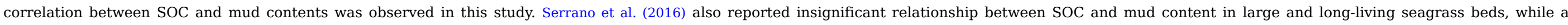

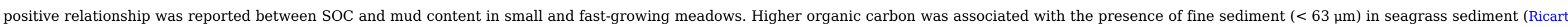

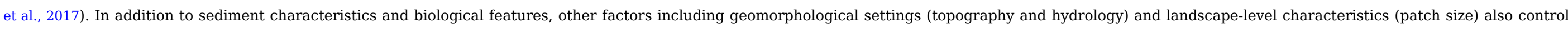

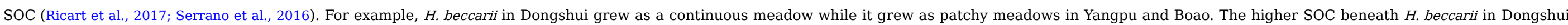

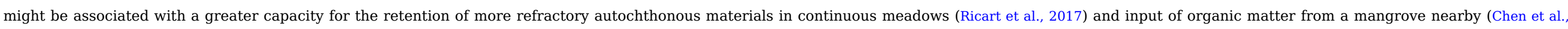
2017).

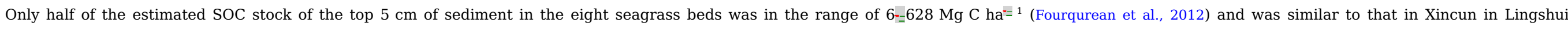

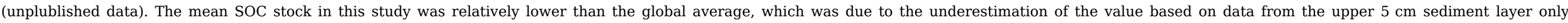

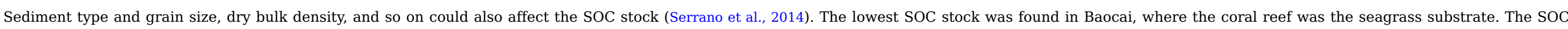

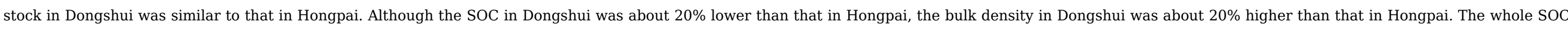

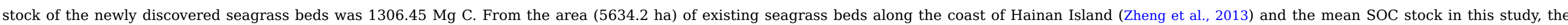
overall SOC stock of seagrass beds in Hainan Province was $40858.5 \mathrm{Mg}$ C (the upper $5 \mathrm{~cm}$ sediment).

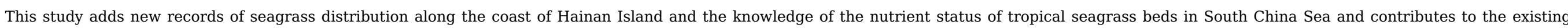

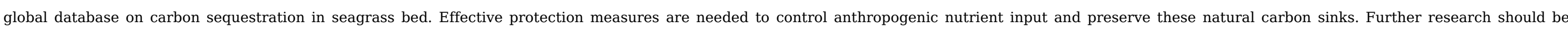

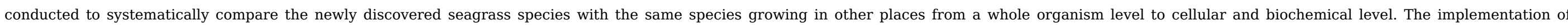
molecular biology techniques is also urgently required to discover new seagrass species in a rapidly changing world.

\section{Acknowledgments}

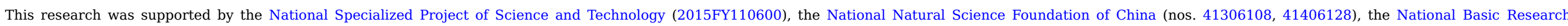

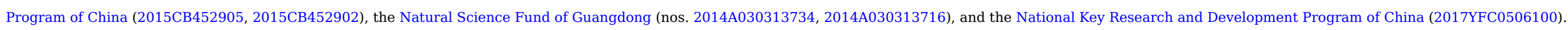

\section{Appendix A.Appendix A. Supplementary data}

Supplementary data to this article can be found online at http://dx.doi.org/10.1016/j.marpolbul.2017.07.066.

\section{References}

Apostolaki E.T., Holmer M., Marbà N. and Karakassis I., Metabolic imbalance in coastal vegetated (Posidonia oceanica) and unvegetated benthic ecosystems, Ecosystems 13, 2010, 459-471.

Barrón C. and Duarte C.M., Dissolved organic matter release in a Posidonia oceanica meadow, Mar. Ecol. Prog. Ser. 374, 2009, 75-84.

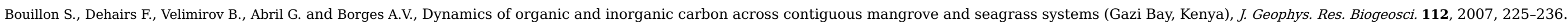
Campbell J., Lacey E., Decker R., Crooks S. and Fourqurean J., Carbon storage in seagrass beds of Abu Dhabi, United Arab Emirates, Estuar. Coasts 38, 2014, 242-251.

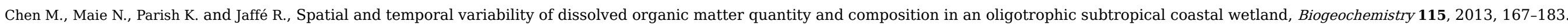

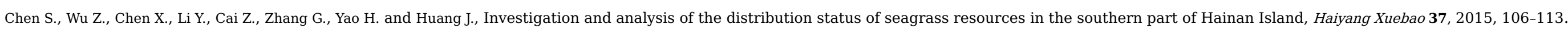

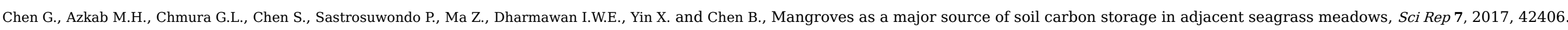


Den Hartog C. and Yang Z., A catalogue of the seagrasses of China, Chin. J. Oceanol. Limnol. 8, 1990, 74-91.

Duarte C.M., Seagrass nutrient content, Mar. Ecol. Prog. Ser. 6, 1990, 201-207.

Duarte C.M., Kennedy H., Marbà N. and Hendriks I., Assessing the capacity of seagrass meadows for carbon burial: current limitations and future strategies, Ocean Coast. Manag. 83, $2013,32-38$.

Fakhrulddin I., Sidik B.J. and Harah Z.M., Halophila beccarii Aschers (Hydrocharitaceae) responses to different salinity gradient, J. Fish. Aquat. Sci. 8, 2013 , 462-471.

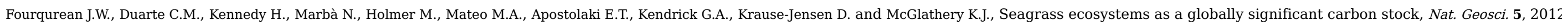
505-509.

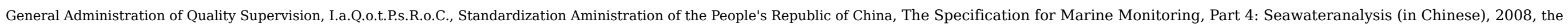
Standards Press of China; Beijing, GB17378.4-2007.

Hemminga M., Hemminga M.A. and Duarte C.M., Seagrass Ecology, 2000, Cambridge University Press.

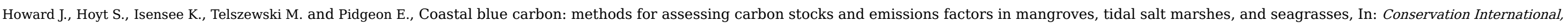
Intergovernmental Oceanographic Commission of UNESCO, International Union for Conservation of Nature, 2014, Arlington; VA, USA..

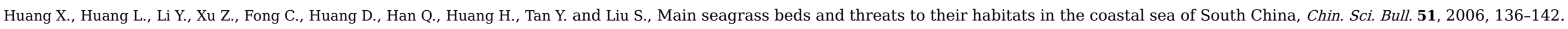

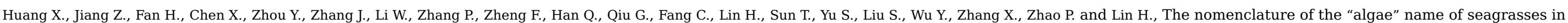
China, Oceanol. et Limnol. Sin. 47, 2016, 290-294.

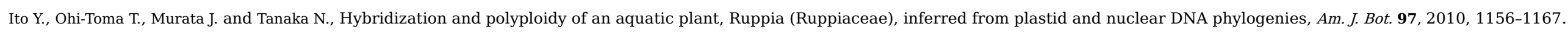

Jiang K., Population Genetic Structure and Gene Flow in the Seagrass Zostera japonica (Zosteraceae) Along the Coastal Regions of South China, 2012, East China Normal University; Shanghai.

Jiang Z., Huang X. and Zhang J., Effect of nitrate enrichment and salinity reduction on the seagrass Thalassia hemprichii previously grown in low light, J. Exp. Mar. Biol. Ecol. 443, 2013, 114-122.

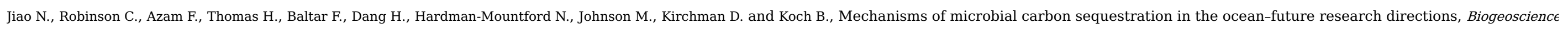

11, 2014, 5285-5306.

Larkum A.W., Orth R.R.J. and Duarte C.M., Seagrasses: Biology, Ecology, and Conservation, 2006, Springer.

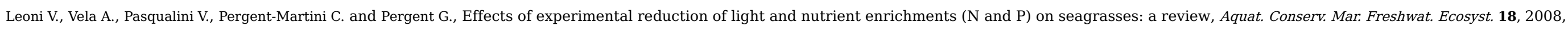
202-220.

Li Q., Huang W. and Zhou Y., A preliminary study of eutrophication and occurrence of red tides in Xincun Harbour, Trans. Oceanol. Limnol. 4, 2010 , 9-15.

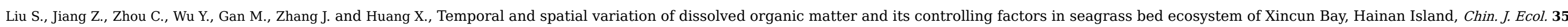
2016, 2144-2151.

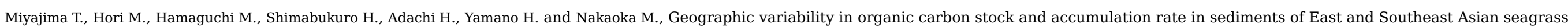
meadows, Glob. Biogeochem. Cycles 29, 2015, 397-415.

Nagata T., Organic Matter-Bacteria Interactions in Seawater, 2008, Microbial Ecology of the Oceans; Second Edition, 207-241.

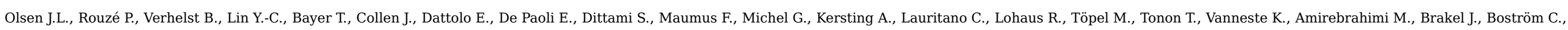

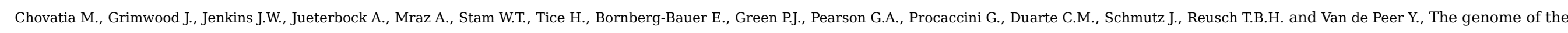
seagrass Zostera marina reveals angiosperm adaptation to the sea, Nature 530, 2016, 331-335. 


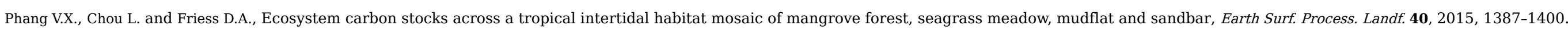
Phillips R.C. and McRoy C.P., Seagrass Research Methods, 1990, Unesco; Paris.

Qiu G., Su Z., Zhong C. and Fan H., Distribution and community characteristics of threatened seagrass Halophila beccarii in Dongzhai Harbo, Hainan, Guihaia 36, 2016 , 882-889.

Ricart A.M., Pérez M. and Romero J., Landscape configuration modulates carbon storage in seagrass sediments, Estuar. Coast. Shelf. Sci. 185, $2017,69-76$.

Serrano O., Lavery P.S., Rozaimi M. and Mateo M.Á., Influence of water depth on the carbon sequestration capacity of seagrasses, Global Biogeochem. Cy. 28, 2014, 950-961.

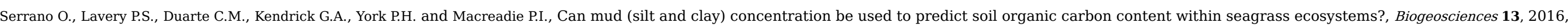
$4915-4926$.

Short F.T. and Coles R.G., Global Seagrass Research Methods, 2001, Elsevier; Amsterdam.

Short F., Carruthers T., Dennison W. and Waycott M., Global seagrass distribution and diversity: a bioregional model, J. Exp. Mar. Biol. Ecol. 350, $2007,3-20$.

Su X., Seagrass Disappear in the Western Coast of Hainan Island, Hainan Daily, 2004, Hainan Daily Press Group; Haikou.

Touchette B.W., Seagrass-salinity interactions: physiological mechanisms used by submersed marine angiosperms for a life at sea, J. Exp. Mar. Biol. Ecol. 350, 2007, 194-215.

Wang D., Wu Z., Chen C., Lan J., Wu R., Chen X., Zhang G. and Li Y., Distribution of seagrass resources and existing threat in Hainan Island, Mar. Environ. Sci. 31, 2012 , 34-38.

Watanabe K. and Kuwae T., How organic carbon derived from multiple sources contributes to carbon sequestration processes in a shallow coastal system?, Glob. Chang. Biol. 21, 2015, 2612-2623.

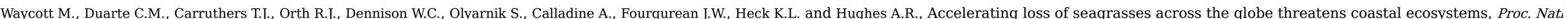
Acad. Sci. 106, 2009, 12377-12381.

Xu N., Clonality and Its Population Genetic and Ecological Consequences in Halophila ovalis (Hydrocharitaceae), 2011, East China Normal University; Shanghai.

Yu S. and den Hartog C., Taxonomy of the genus Ruppia in China, Aquat. Bot. 119, 2014, 66-72.

Zhang J., Huang X. and Jiang Z., Physiological responses of the seagrass Thalassia hemprichii (Ehrenb.) Aschers as indicators of nutrient loading, Mar. Pollut. Bull. 83, 2014, 508-515.

Zheng F., Qiu G., Fan H. and Zhang W., Diversity, distribution and conservation of Chinese seagrass species, Biodivers. Sci. 21, 2013, 517-526.

Ziegler S., Kaiser E. and Benner R., Dynamics of dissolved organic carbon, nitrogen and phosphorus in a seagrass meadow of Laguna Madre, Texas. Bull. Mar. Sci. 75, 2004, 391-407.

\section{Appendix A.Appendix A. Supplementary data}

Multimedia Component 1

Supplementary material

alt-text: Image 1

\section{Highlights}

- Eight new seagrass beds were discovered with an area of 203.64 ha.

- Leaf $\mathrm{N}$ content and C:N ratio indicated $\mathrm{N}$ is in replete status in seagrass bed.

- Lower leaf $\mathrm{C}$ content of seagrass was observed in seagrass bed with higher nutrient. 
- The mean SOC stock was $7.02 \pm 3.57 \mathrm{Mg} \mathrm{C}^{-1}$, with the whole as $1306.45 \mathrm{Mg} \mathrm{C}$.

\section{Queries and Answers}

Query:

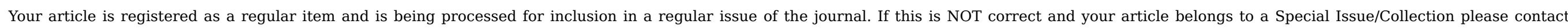
l.barker@elsevier.com immediately prior to returning your corrections.

Answer: Yes

Query:

Please confirm that given names and surnames have been identified correctly and are presented in the desired order, and please carefully verify the spelling of all authors' names.

Answer: Yes

Query:

The author names have been tagged as given names and surnames (surnames are highlighted in teal color). Please confirm if they have been identified correctly.

Answer: Yes

Query:

Supplementary caption was not provided. Please check the suggested data if appropriate, and correct if necessary

Answer: it is necessary and correct 